Jurnal

Kardiologi Indonesia

J Kardiol Indones. 2015;36:52-6I

ISSN 0I 26/3773

\title{
The Role of Cardiac Computed Tomography in Tetralogy of Fallot and Pulmonary Atresia
}

\author{
Oktavia Lilyasari
}

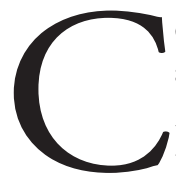

ongenital heart disease (CHD) occurs in about 6 to 8 of 1000 live births with the increasing prevalence can be attributed to major improvements in diagnosis and treatment. Imaging has an important role in diagnosis of CHD. It outlines anatomy and physiology, helps to refine management, evaluates the consequences of interventions and helps guide prognosis. However, no single available imaging modality fulfills these roles for all patients and diseases. Therefore, assessment for CHD must involve multimodalities that can be used in a complementary fashion, and that together are sensitive, accurate, reproducible, and cost effective, whilst minimizing harm.

In previous years, the diagnosis and the treatment of congenital malformations have often depended on cardiac catheterization. In many institutions, cardiac catheterization still remains the gold standard against which other modalities are measured. In the past decade, however, imaging methodologies have increasingly shifted toward the use of less invasive and noninvasive techniques. Although echocardiography is the most commonly used imaging modality for diagnosis and follow-up of subjects with CHD, the evolution of cardiovascular magnetic resonance (MR) imaging and increasingly computed tomography (CT)

\footnotetext{
Alamat Korespondensi

Dr. Oktavia Lilyasari, Department Cardiology and Vascular Medicine, Faculty of Medicine Universitas Indonesia. National Cardiovascular Center Harapan Kita Jakarta. E-mail: oktavia_lilyasari@ yahoo.com
}

does offer new ways to visualize the heart and the great vessels.

A scientific statement from American Heart Association in 2011 recommended that complete cardiac echocardiographic imaging or alternative noninvasive imaging modes such as MRI or CT are indicated before invasive cardiac catheterization to facilitate planning of data collection and performance of an intervention (Class I Level of Evidence: A). Diagnostic catheterization is no longer indicated in the routine preoperative evaluation of most congenital defects, to prevent unnecessary risk and exposure to radiation. Currently, catheter-based angiography is reserved for endovascular intervention and obtaining direct hemodynamic measurement.

In this review, we will discuss the role of cardiac computed tomography in Tetralogy of Fallot (TOF) and Pulmonary atresia (PA) which is a new adjunctive technique that currently available at our center besides echocardiography and cardiac catheterization.

\section{A. Tetralogy of Fallot}

Tetralogy of Fallot is one of the most common congenital heart malformations. It represents $6.8 \%$ of all CHD. The embryology of TOF is thought that misalignment of the conal septum in relation to the ventricular endocardial cushion leads to a lack of stimulus for the development of the membranous septum. It is comprising a ventricular septal defect (VSD), right ventricular outflow tract (RVOT) 
obstruction, right ventricular hypertrophy and an overriding aorta. Disease severity of presentation depends on the degree of RVOT obstruction.

TOF represents a spectrum of disease and is

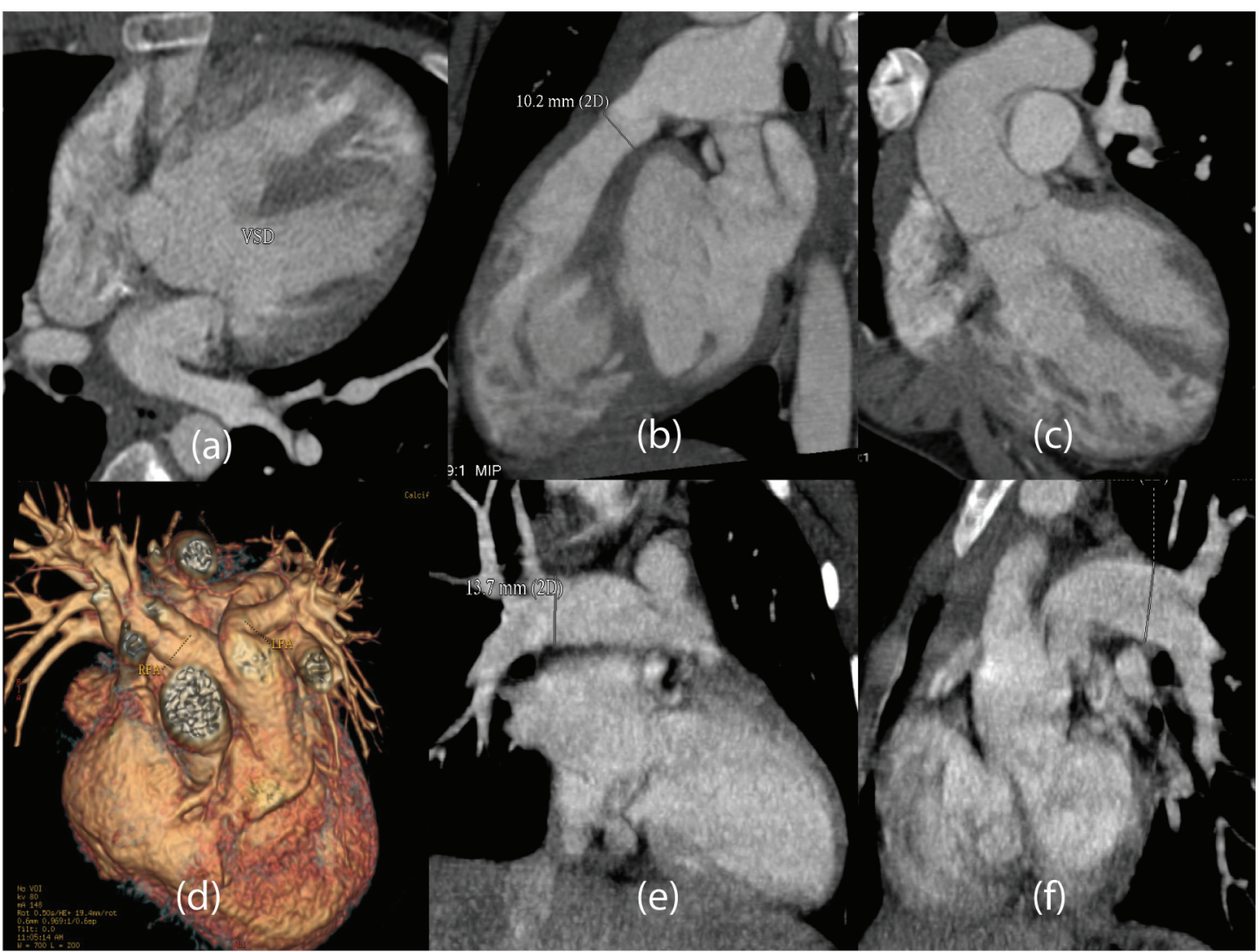

Figure 1. The CT images of patient withTOF. The MIP images showed sub aortic VSD (a,c), infundibular pulmonary stenosis with right ventricle hypertrophy (b) and overriding of the aorta (c). The volume rendering (d) and MIP (e,f) below showed the confluence PA and the sizing of PA branches

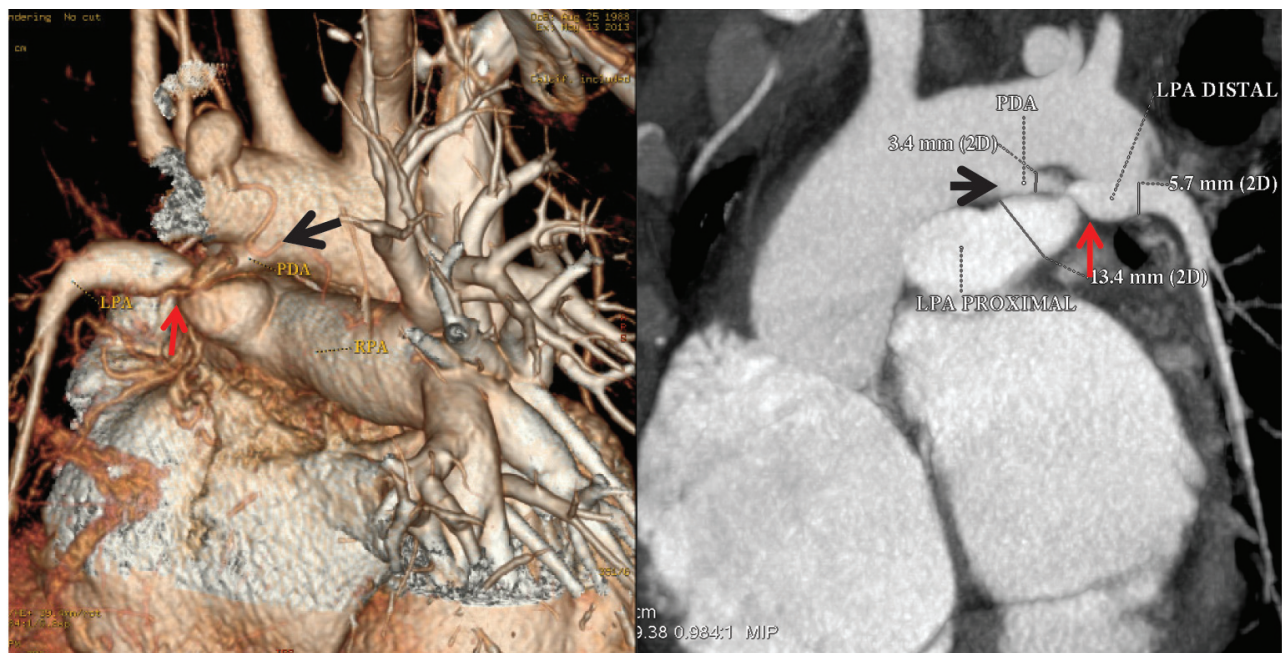

Figure 2. CT images of a patient with TOF, PDA, with hypoplastic distal LPA. The volume rendering and MIP images revealed PDA (black arrow) and stenosis at the mid part of LPA (red arrow) at the drainage of PDA. Distal LPA is hypoplastic. 
present in 3 major variants: TOF with pulmonary valve or RVOT stenosis, TOF with pulmonary atresia, and TOF with absent pulmonary valve. TOF with pulmonary atresia is the most severe form and accounts for $20 \%$ of the TOF population.

\section{Objectives of CT examination in TOF}

- Assess RVOTO or fixed narrowing of the sub- pulmonary infundibulum

- Assess pulmonary valve morphology

- Assess Pulmonary artery's confluence, caliber and branches

- Identify any additional VSDs

- Identify any PDA

- Look for aortic root dilatation

- Look for Collateral or MAPCAs

- Look for coronary artery anomalies

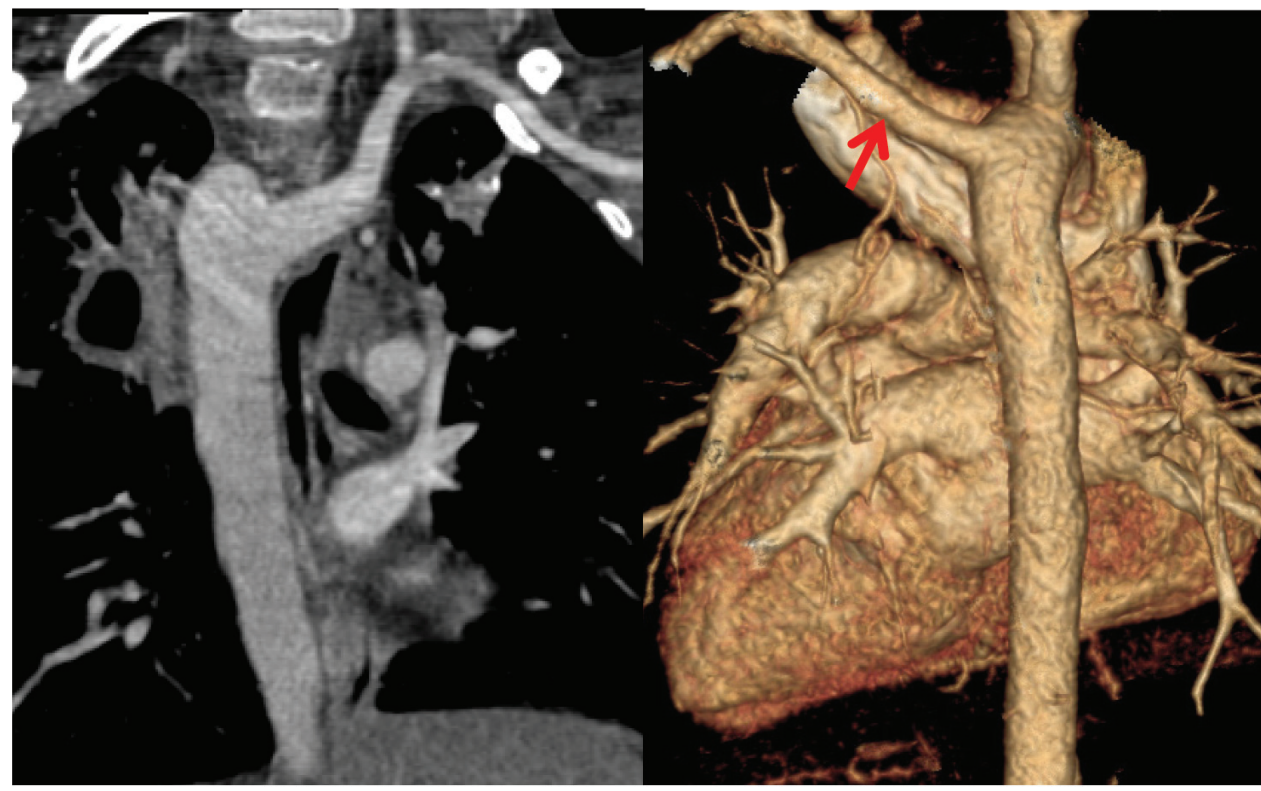

A

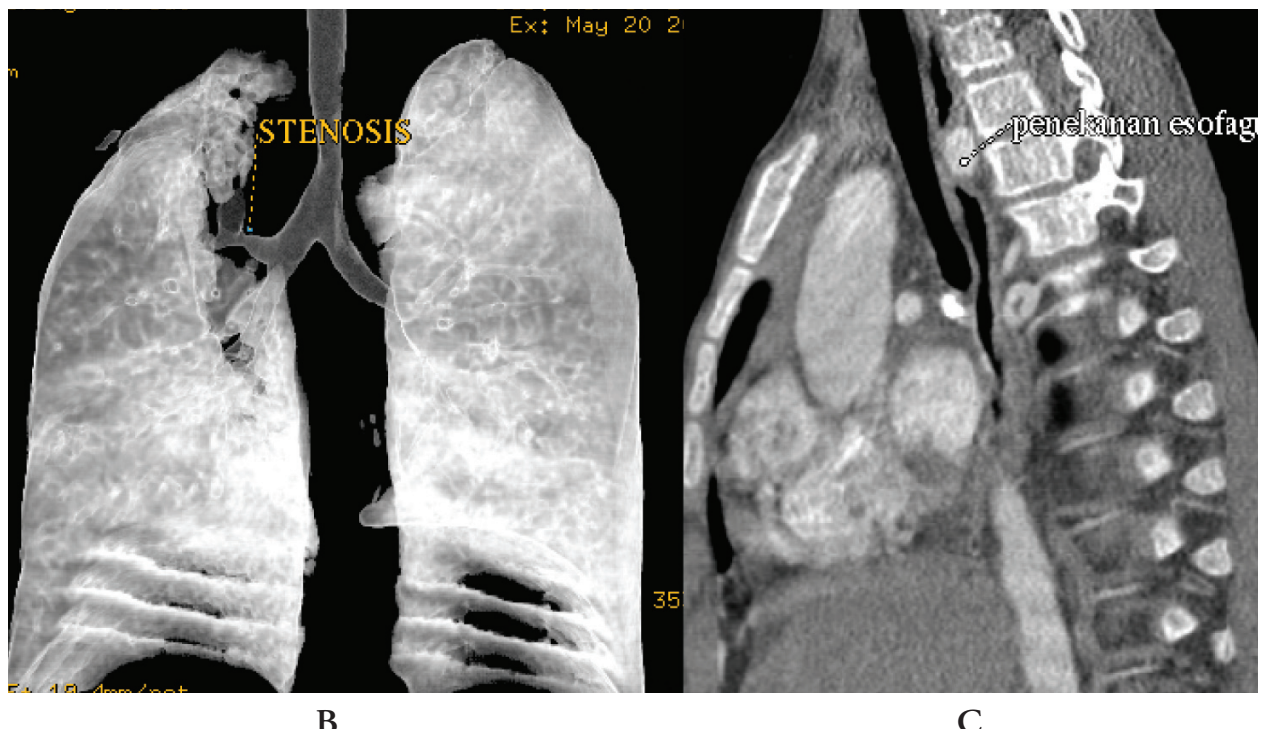

B

$\mathrm{C}$

Figure 3. The MIP and volume rendering CT images of patient with TOF showed the right aortic arch with abberant left subclavian artery (LSCA) which cause compression of the right upper branch bronchi (b) and the esophagus (c) 


\section{Tetralogy of Fallot with aortic arch anomalies}

Anomalies in the developmental process of the aortic arch and its main branches can form vascular rings around the trachea and esophagus that may or may not be symptomatic depending on the location and degree of compression. Vascular ring is an anomaly in which the tracheal and esophagus (or its atretic remnant) are completely surrounded by vessel. Anomalies of aortic

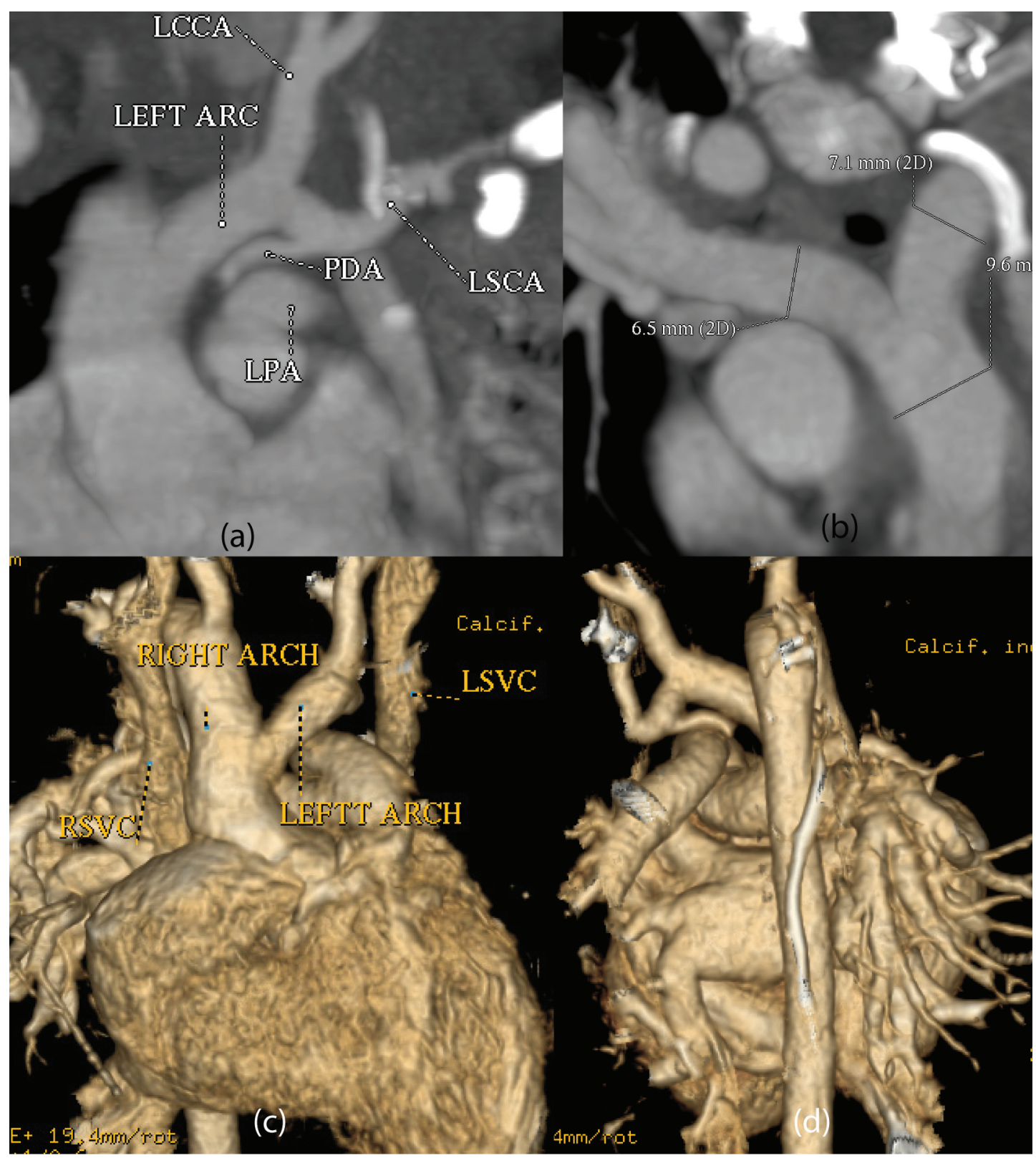

Figure 4. The CT images of patient with TOF and double aortic arch and atresia distal left aortic arch segment. The MIP images (a,b) and volume rendering (c, d) CT images revealed TOF with confluence pulmonary artery, double aortic arch ( Right sided arch forms right common carotis artery (RCCA), right subclavian artery (RCCA) and descending aorta (AoD), the left sided aortic arch forms left common carotis artery (LCCA), lef subclavian artery (LSCA) and small patent ductus arteriosus (PDA) to the left pulmonary artery (LPA) with atresia of distal left arch) 
arch may present in patients with Tetralogy of Fallot and are common incidentally findings during standard diagnostic imaging.

\section{TOF with Infective Endocarditis}

Adult patients with Tetralogy of Fallot carry a high risk for the development of infective endocarditis (IE). Pulmonary artery aneurysm is a vascular abnormality morphologically characterized by focal dilatation of the vessel involving all three layers of the vessel with or without mural thrombi or wall calcification.
Mycotic aneurysms caused by bacteria are usually associated with IE and septic embolism. Figure 3 indicates an IE in adult with TOF may involve more than one valve simultaneously and may cause mycotic pulmonary artery aneurysm. It is important to early recognize this abnormality because of its association with higher risk of rupture and may cause fatal and serious complication in adult CHD. Multi-detector CT angiography provides useful information regarding size, number, location and extent of the aneurysm and can help to detect any vascular abnormalities in adult CHD with IE.

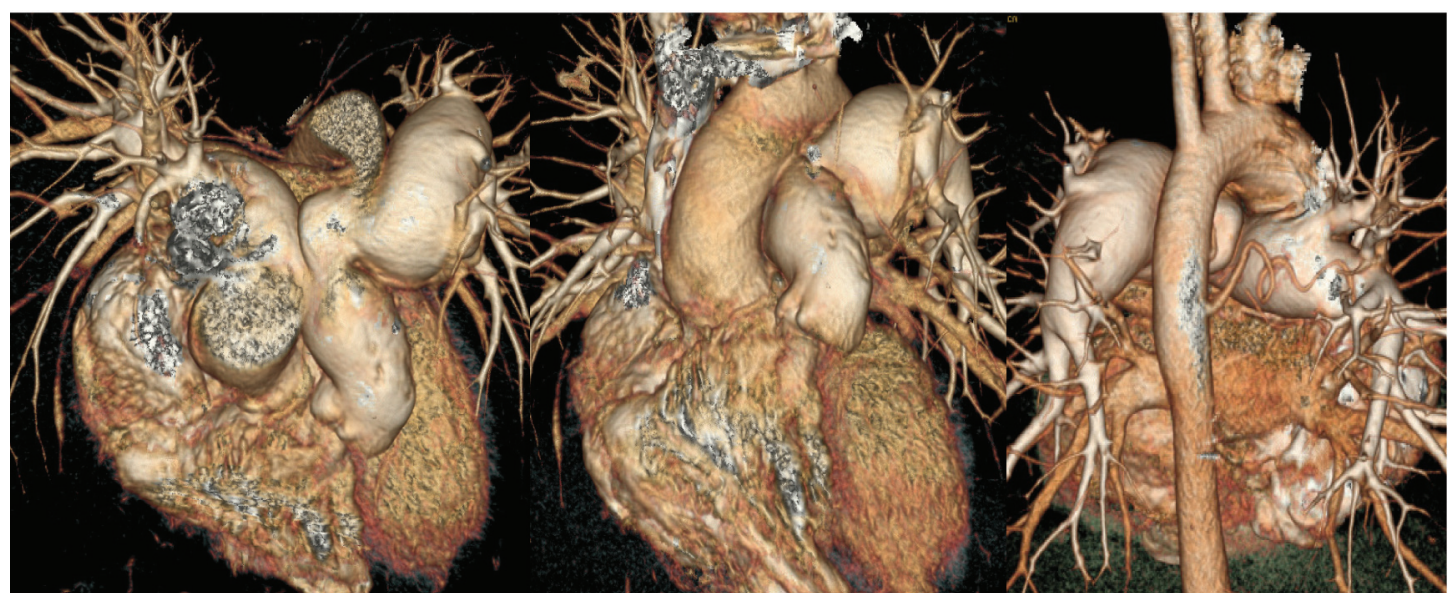

Figure 5. The CT images of a woman with Tetralogy of Fallot and infective endocarditis. The volume rendering images showed a huge pulmonary artery caused by pulmonary mycotic aneurysm

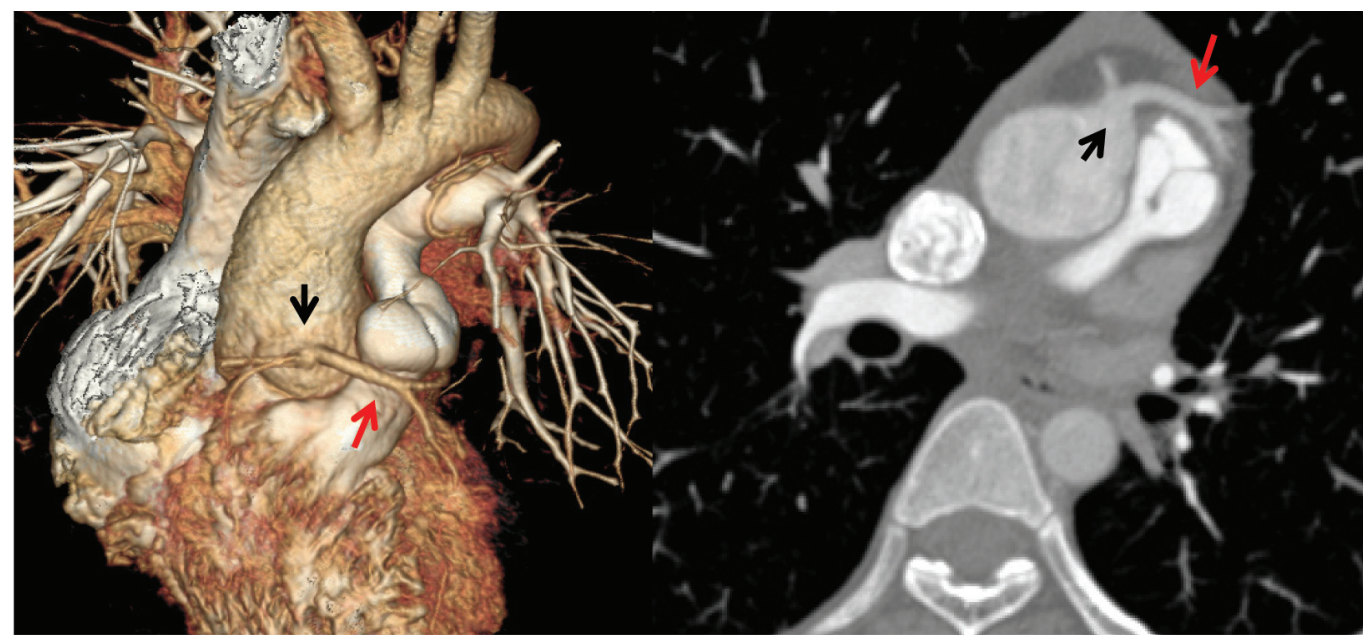

Figure 6. The CT images of TOF patiet with coronary artery anomalies. The left anterior descending (LAD) coronary artery (black arrow) arises from the right right sinus and coursing anterior to the RVOT (red arrow). 


\section{TOF with coronary artery anomalies}

Coronary anomalies are common in TOF, occurring 2 up to $14 \%$ of patients with TOF. Anomalies in the origin and course of the coronary arteries that involved the right ventricle anterior wall are highly significant. The most common anomaly is an anomalous left anterior descending (LAD) coronary artery arising from the right coronary artery or right sinus of valsava and coursing anterior to the RVOT (figure 5). The anomalous of coronary artery anomalies must be identified preoperatively to prevent inadvertently damaged during repair.

\section{B. Pulmonary atresia}

Patients with pulmonary atresia represent a more complicated population. The pulmonary artery (PA) can range from normal in size to complete atresia of the central pulmonary arteries. In some cases of pulmonary atresia, the lungs are supplied by patent ductus arteriosus
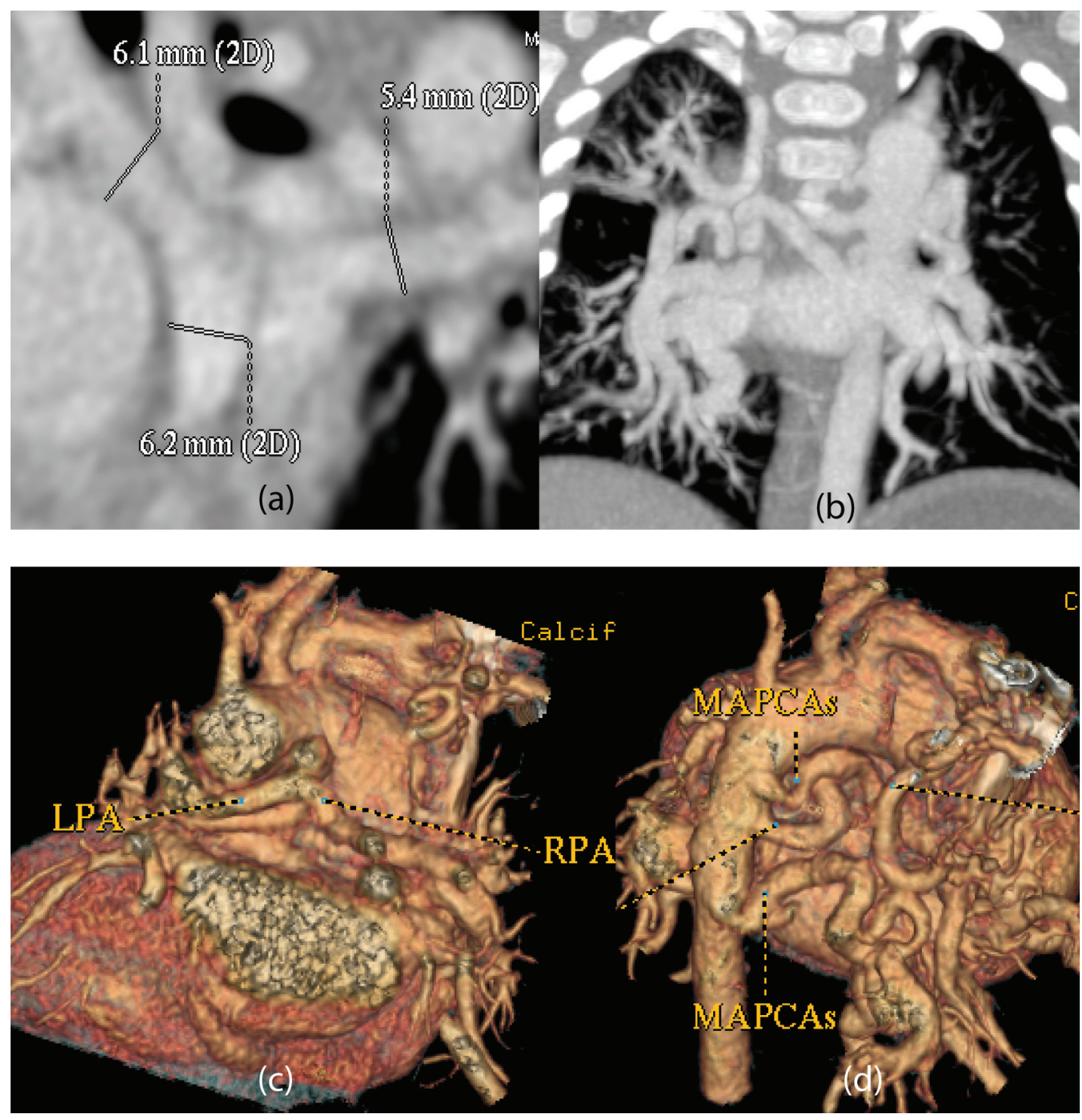

Figure 7. MIP and Volume Rendering CT images of patient with PA, VSD, MAPCAs showed confluence pulmonary arteries that were supplied from MAPCAs $(\mathrm{a}, \mathrm{b}, \mathrm{c}, \mathrm{d})$ and higher arborization of the right lung due to unbalanced blood flow distribution from the MAPCAs (b) 
(PDA) or systemic collateral from the aorta or its branches, called multiple aorto-pulmonary collateral arteries (MAPCAs). These may represent the only blood supply to portions of the lung or may be redundant to existing pulmonary arteries. In patients with extensive collateral the procedures such as collateral embolization with device or ligation of collateral and unifocalization of collateral may be performed.
Ideally, the cardiac catheterization is a gold standard to define the blood supply to the each portion of the lung. But in some cases we could not find the native PA during cardiac catheterization examination. In those particular cases, the cardiac CT examination will be useful to add some information regarding the availability of the native PA, other blood supply resources, and the relation of the MAPCAs with other structures.

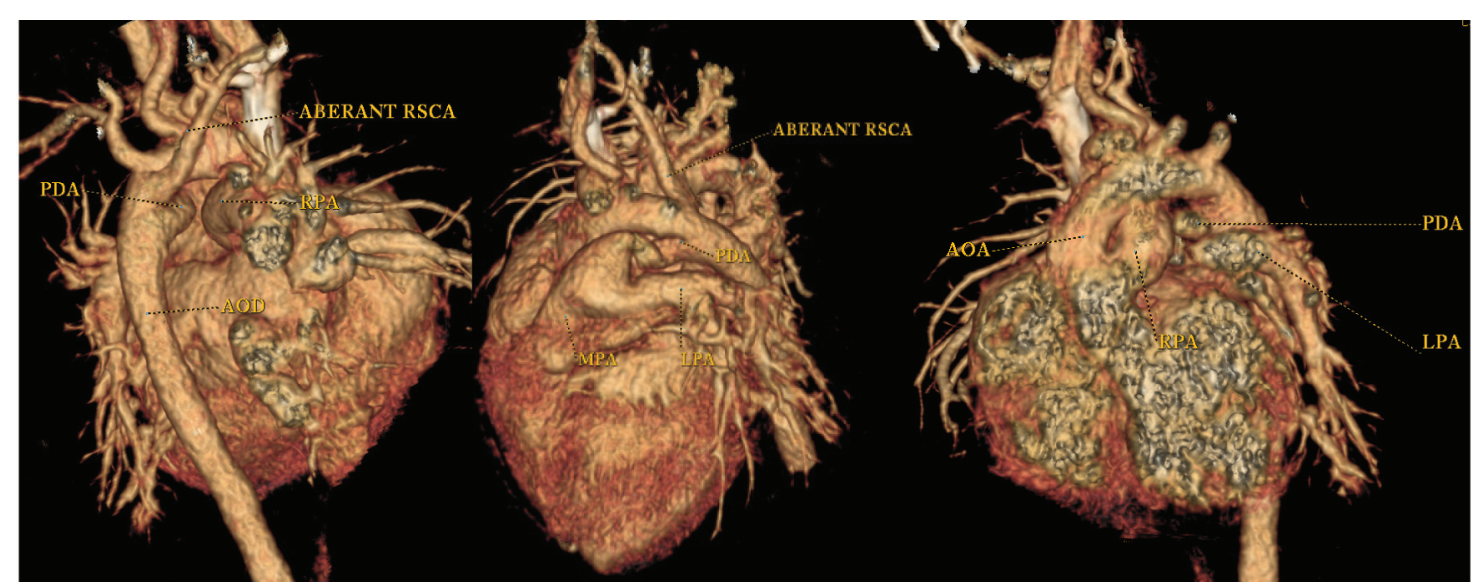

Figure 8. The volume rendering CT images of patient with Pulmonary Atresia, Sub aortic VSD showed atretic of pulmonary valve with non-confluence pulmonary arteries. Right pulmonary artery arises from aorta (AORPA), MPA arises from PDA, and the abberant right subclavian artery persists.

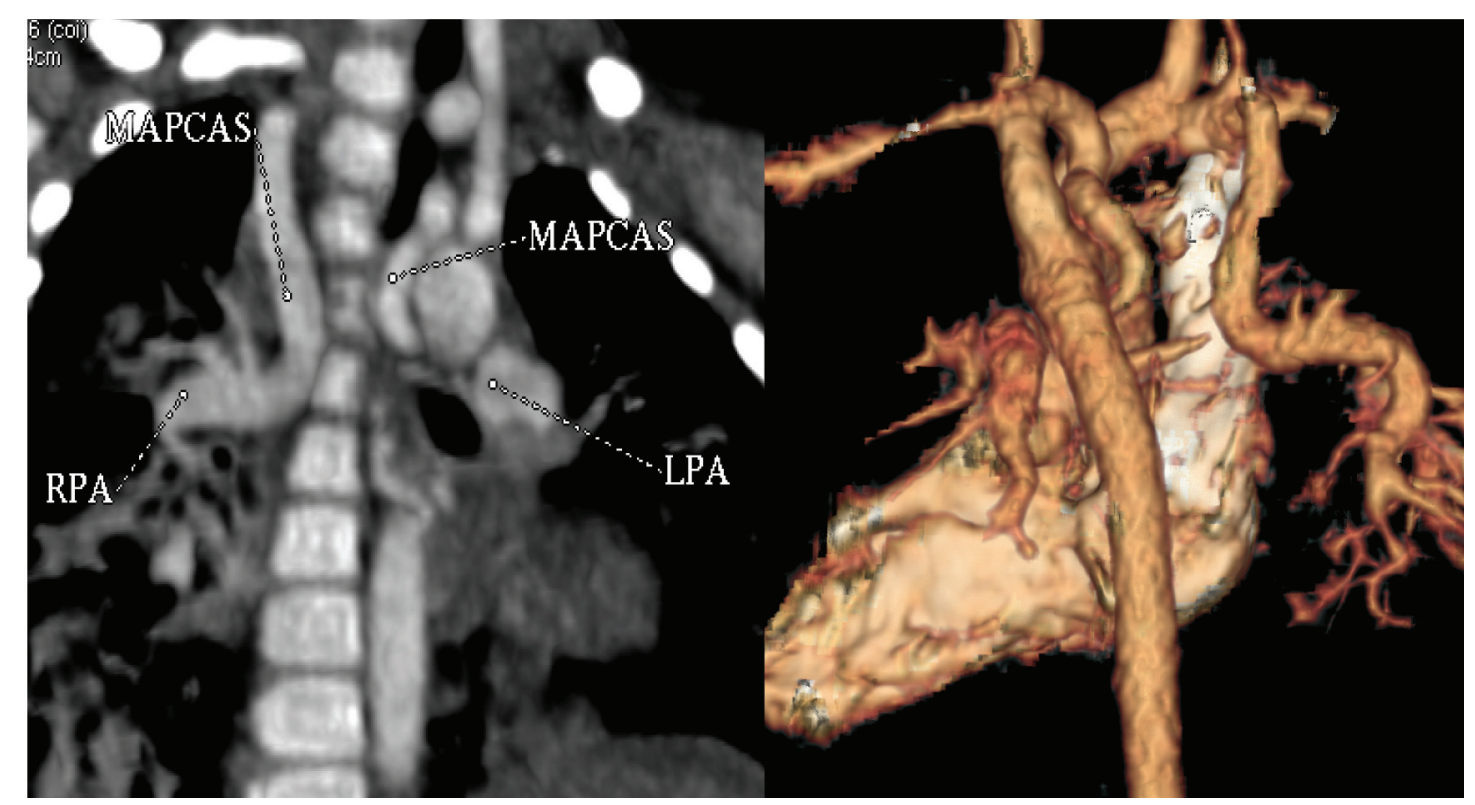

Figure 9. The CT images of patient with PA, VSD, MAPCAs. The MIP and volume rendering CT images showed non-confluence pulmonary arteries. The blood supply for RPA comes from right sub clavian artery (RSCA) and the blood supply for the LPA comes from the left subclavian artery (LSCA) 


\section{Objectives of CT examination}

- Characterize the presence or absence of native Pas (supplied by PDA or BT shunt)

- Assess native Pulmonary artery's confluence, caliber and branches

- Delineate number and course of multifocal pulmonary blood supply (MAPCAs

- Show relationship of MAPCAs to other structures: trachea, bronchi, oesophagus

- Evaluate the completeness of pulmonary vascular arborization of both lung

\section{Pulmonary atresia with confluence pulmonary artery \\ Pulmonary Atresia with Non confluence PA}

\section{MAPCAs and collaterals}

\section{Post-operative evaluation}

Complete repair are commonly performed on infant upper 6 month of age. Some patients who have inadequate pulmonary artery anatomy or coronary

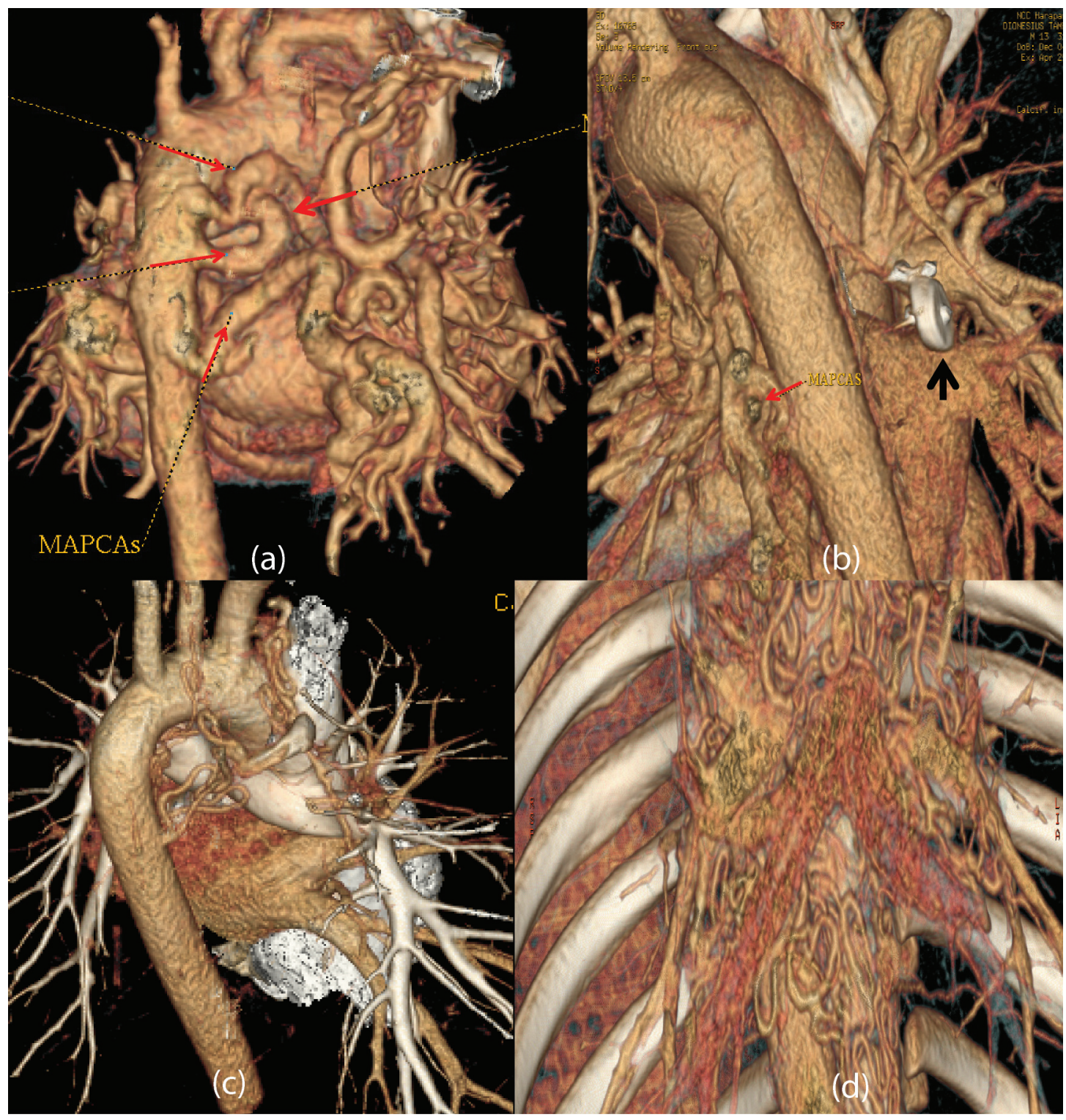

Figure 10. The volume rendering CT images showed MAPCAs (red arrow)(a,b). Device embolization of the MAPCAs (black arrow)(b), and Collaterals (bellow) 

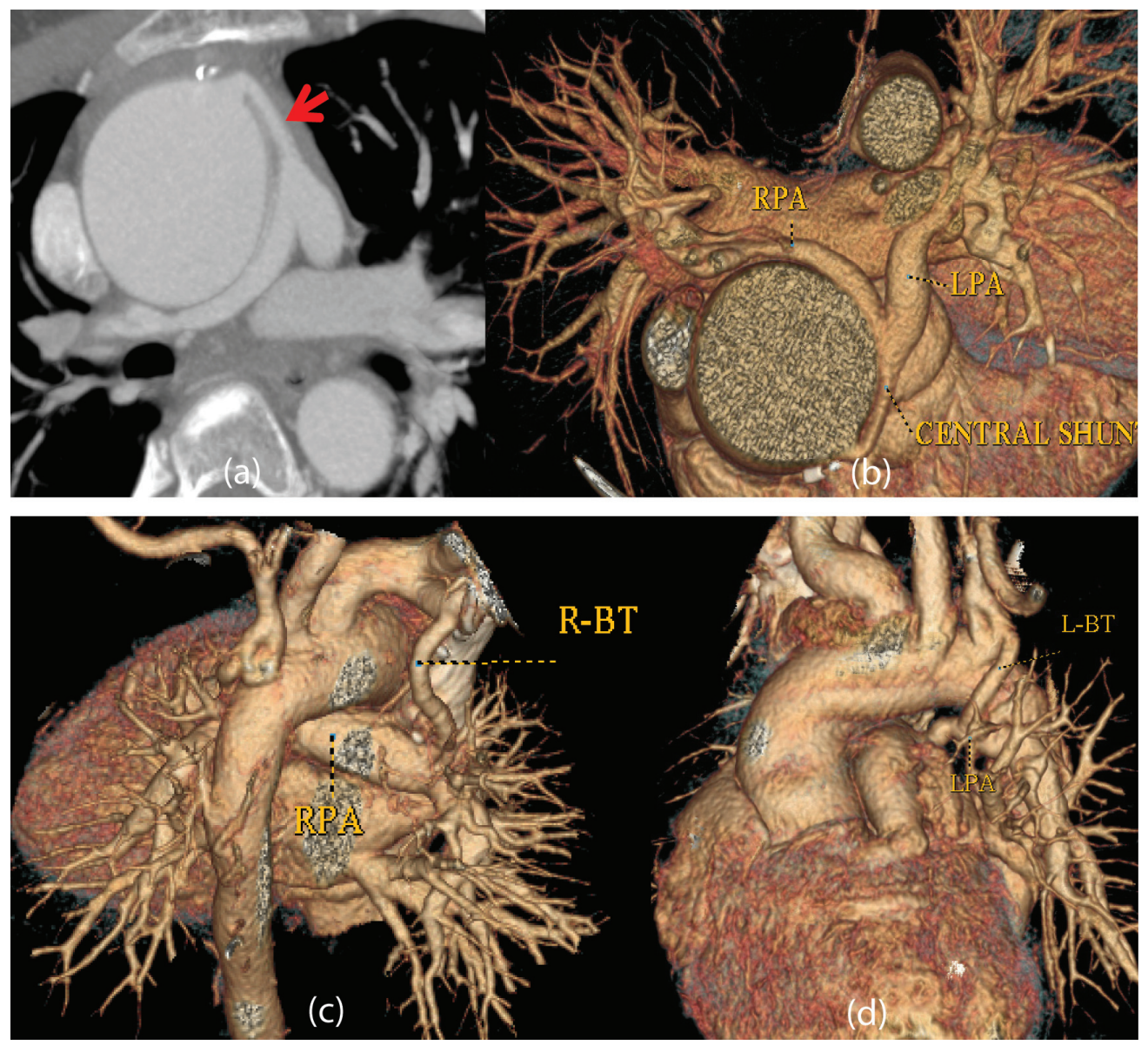

Figure 11. The MIP and Volume rendering images of patient after BT shunt procedure showed patent central shunt $(\mathrm{a}, \mathrm{c})$, patent right BT Shunt (c) and Left BT shunt (d)

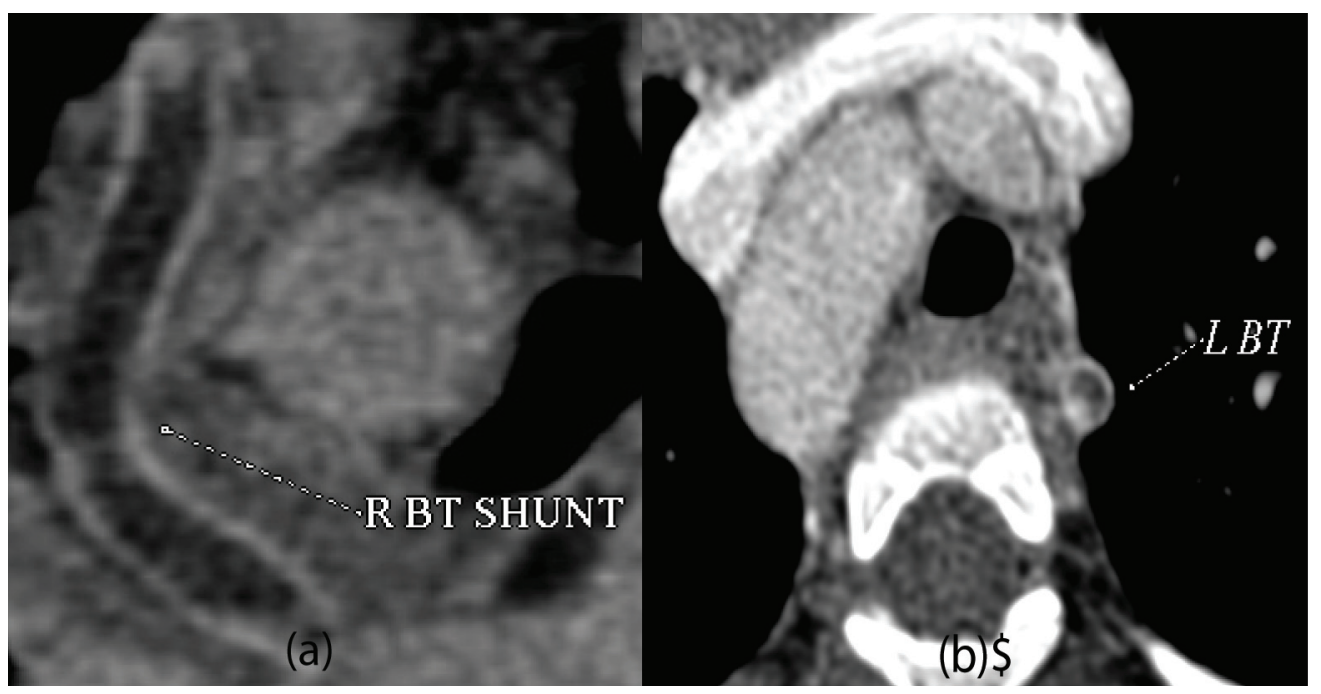

Figure 12. The MIP images of patient after BT shunt procedure revealed Non-patent R-BT (a) and L-BT shunt (b) 


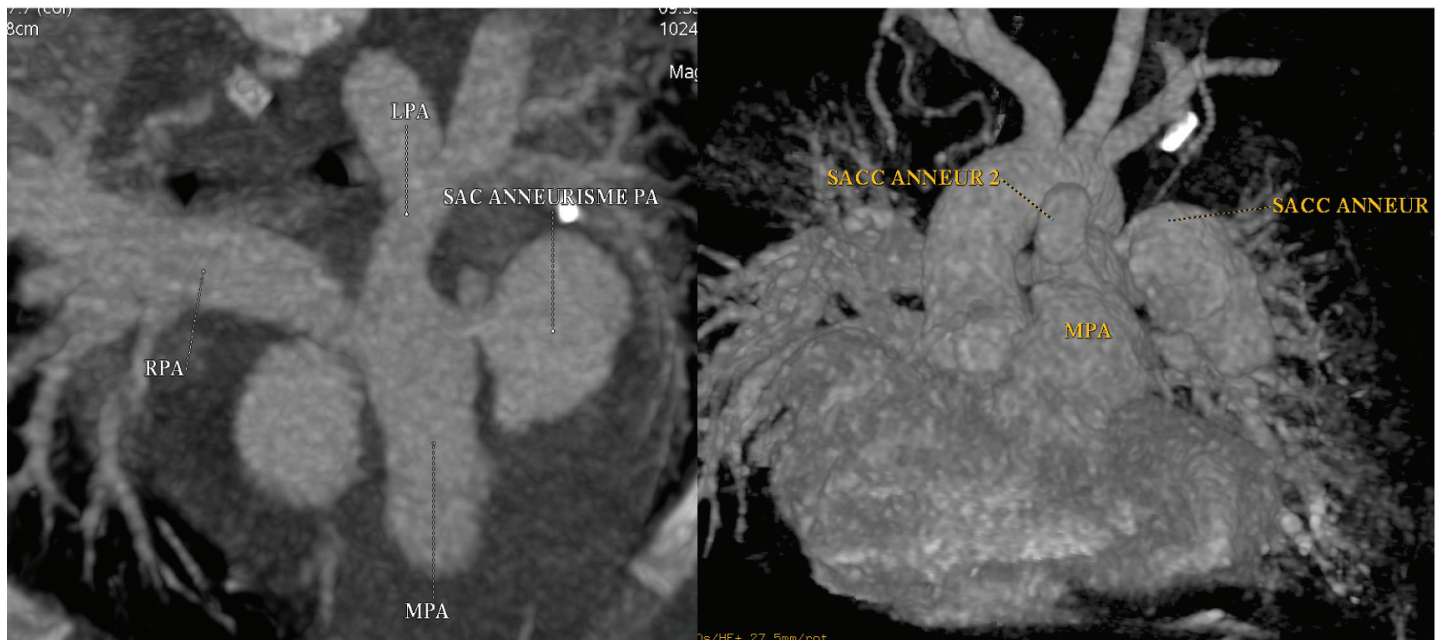

Figure 13. The MIP and volume rendering images of patient with post correction of TOF who suffered severe infection revealed saccular aneurysm of MPA and LPA

artery anomalies may require a staged procedure. Surgical palliation for TOF began in the 1940 with the performance of the first Blalock-Taussig shunt.

\section{Conclusions}

Imaging plays an important role in the diagnostic and management of Tetralogy of Fallot and pulmonary atresia. It helps the doctors decide the type of operation. Despite ionizing radiation and iodinated contrast needed, the CT has the advantage of short scanning times, less sedative and wide availability. The use of cardiac CT in TOF has to be concerned to provide additional anatomy information to guide the management of patient with TOF and pulmonary atresia.

\section{Aknowledgement}

I would like to thank all the team of Cardiac CT in National Cardiovascular Center Harapan Kita Jakarta for their contributions in providing all the $\mathrm{CT}$ images in this review.

\section{References}

1. Goo H.W., Park I.S., Ko J.K., Kim Y.H., et al.. CT of Congenital Heart Disease : Normal anatomy and typical pathologic Conditions. Radiographics 2003;23:S147- 164.

2. Dillman JR. Hernandez E.J. Role of CT in the evaluation of Congenital Cardiovascular Disease in Children. American Journal Radiology 2009;192:1219-1231.

3. Ou P., Celermajer D S., Calcagni G., et al. 3Diensional CT Scanning : a new diagnostic modality in congenital heart disease. Heart 2007; 93:908-913

4. Gilkeson C. CT evaluation of adult congenital heart disease " a practical approach.Supplement to apllied radiology, Dec 2006. www.appliedradiology.com

5. Goo H W. State of the Art CT imaging Technic for Congenital Heart Disease. Korean J Radiol 2010;11: 4-18.

6. Garner R.D., Sutton N.J., Weinstein S., Franco H.S., Haramati L.B. MRI and computed tomography of cardiac and pulmonary complications of Tetralogy of Fallot in adults. J Thorac Imaging 2010;25:183-190

7. Kervancioglu M., Tokel K., Varan., Yildirim S.V. Frequency, orgins and courses of anomalous coronary artery in 607 Tuekish Children with Tetralogy of Fallot. Cardiol J 2011;18,5:54651. 